\title{
La Costituzione di Licurgo secondo Lévesque: un «ritratto» della Francia pre-rivoluzionaria
}

\author{
Marcello TozzA \\ Universidad de Málaga \\ marcello.tozza@virgilio.it
}

Recibido: 8-05-2012

Aceptado: 6-12-2013

\begin{abstract}
RESUMEN
Las leyes de Esparta ofrecen a Lévesque un término de comparación con el sistema político francés anterior a la Revolución: aunque el estudioso condene abiertamente la estructura del feudalismo medieval, alude también a aquellos aspectos políticos y sociales que sobreviven a la muerte jurídica del feudalismo, hasta desaparecer totalmente por obra de la Revolución. Además, criticando Esparta, Lévesque se muestra en contra de la política de los Montañeses, que utilizaron el ordenamiento espartano como base ideológica sobre que imaginar la nueva sociedad francesa, llevando el país a una dictadura de pocos «iguales» que representa una vuelta atrás con respecto a los ideales de la Revolución.
\end{abstract}

Palabras clave: Lévesque, Licurgo, Esparta, Revolución francesa.

\begin{abstract}
The laws of Sparta offer to Lévesque a term of comparison with the political system existing before the French Revolution: the scholar openly condemns the structure of medieval feudalism, but also he refers to those political and social aspects that survive the legal death of feudalism, completely disappearing only with the Revolution. Furthermore, criticizing Sparta, Lévesque expresses his disappointment toward the policy of the Montagnards, who used the Spartan system as an ideological basis on which they constructed the new French society, leading the country to a dictatorship of few «equals» that looks like turning back with respect to the ideals of the Revolution.
\end{abstract}

Keywords: Lévesque, Lycurgus, Sparta, French Revolution.

In un dizionario biografico, curato dallo studioso François-Xavier de Feller fin dagli anni ' 80 del secolo XVIII, e pubblicato nel 1849 con il titolo definitivo di Biografia universale, o dizionario storico degli uomini che si sono fatti un nome, si dice di Pierre-Charles Lévesque che «fu legato ai filosofi, di cui condivideva i sentimenti» ${ }^{1}$.

${ }^{1}$ «Il fut lié avec les philosophes, dont il partagea les sentiments» (DE FeLLER, François-Xavier (1849), V, p. 239). 
Effettivamente, leggendo la sua opera sulla vita e le massime dei filosofi greci, vi sono diversi punti in cui Lévesque sembra non poter evitare di esprimere la propria partecipazione emotiva alle vicende dei personaggi su cui si incentra la trattazione; l'esempio più lampante appare quello della biografia di Socrate, a cui l'autore dedica un ampio spazio, mostrandosi senza dubbio affascinato dall'attività speculativa del pensatore: parlando della condanna a morte del filosofo, che aveva come capi d'accusa l'empietà e la corruzione dei giovani, Lévesque non nasconde il proprio risentimento per il fatto storico, definendo Socrate «uno degli uomini antichi dai costumi più puri, forse il più religioso dei filosofi», ed ancora «martire della filosofia ... che muore per delle opinioni» ${ }^{2}$.

Tuttavia, se nella trattazione di argomento filosofico il coinvolgimento appare di carattere squisitamente emotivo, nell'ambito storico-politico Lévesque sembra utilizzare i testi antichi, oggetto d'analisi, come mezzo per evidenziare dinamiche socio-culturali appartenenti a realtà storiche molto più vicine alla sua epoca.

Nell'introdurre la sua edizione dei Detti dei Lacedemoni di Plutarco, lo studioso menziona il legislatore Licurgo, personaggio a cavallo tra storia e mito, servendosi della sua vicenda come spunto da cui trarre una rilevante riflessione di carattere storiografico:

Licurgo, si dice, stabilì a Sparta il regno dell'uguaglianza, divise le terre ugualmente tra i cittadini, e fece degli Spartani un popolo di fratelli. Quest'elogio è bello, senza dubbio; si ammira il grande uomo che ne è l'oggetto: ma quando si esaminano i fatti, si vede che Licurgo non stabilì a Sparta altro che la più oppressiva aristocrazia ... Il piccolo numero di uomini privilegiati che portavano il nome di Spartiati, i soli che godevano dei vantaggi della repubblica, sembra che non fosse mai arrivato a più di diecimila ... I semplici Laconici erano in numero di gran lunga superiore, e non avevano alcun diritto alla cittadinanza ... Sparta offriva dunque il ritratto di ciò che si vide in Francia sotto la prima e la seconda dinastia, allorquando una casta non numerosa e privilegiata attribuiva solo a sé stessa il nome di popolo francese, aveva essa sola l'accesso alle pretese assemblee della nazione, ed essa sola prendeva parte all'elaborazione delle leggi, mentre la grande massa del popolo francese, designata con i nomi di coloni, dipendenti, contadini, servi, soffriva in modi diversi una graduale servitù ${ }^{3}$.

${ }^{2}$ «Un des hommes de l'antiquité qui parôit avoir eu les moeurs les plus pures, celui des philosophes qui fut peut-être le plus religieux», «martyr de la philosophie ... qui meurt pour des opinions» (LÉVESQUE, Pierre-Charles (1795), pp. 48, 49, 53).

${ }^{3}$ «Lycurgue, dit-on, établit à Sparte le regne de l'égalité, partagea les terres également entre les citoyens, et fit des Spartiates un peuple de frères. Cet éloge est beau, sans doute; on admire le grand homme qui en est l'objet: mais quand on examine les faits, on voit que Lycurgue n'établit à Sparte que l'aristocratie la plus oppressive ... Le petit nombre d'hommes privilegiés qui portoient le nom de Spartiates, et qui seuls jouissoient des avantages de la république, paroît n'avoir monté jamais à plus de dix mille ... Les simples Laconiens étoient en bien plus grand nombre, et ils n'avoient aucun droit à la cité ... Sparte offroit donc le tableau de ce qu'on vit en France sous la première et la seconde races, lorsqu'une caste pas nombreuse et privilégiée s'attribuoit à elle seule le nom de peuple françois, avoit seule l'entrée aux prétendues assemblées de la nation, et prenoit seule part à la confection des loix, tandis que la grande masse du peuple françois, désignée par les noms de colons, de tributaires, de villains, de serfs, gémissoit différemment dans un servitude graduée» (LÉvesQue, Pierre-Charles (1794), pp. 4-7). 
In effetti, riguardo alla storicità di Licurgo, lo stesso Plutarco dichiara, come premessa alla sua Vita di Licurgo, che su questo legislatore non si può dire nulla di sicuro, poiché sono controverse le notizie sia sulla sua collocazione cronologica che sulle sue attività. Ciò che però appare chiaro è l'indiscussa attribuzione allo stesso Licurgo, da parte della tradizione classica, di una serie di riforme che posero fine all'ordinamento monarchico assoluto a Sparta: Plutarco pone un forte accento sull'importanza dell'istituzione della $\gamma \varepsilon \rho o v \sigma i ́ \alpha$, il consiglio composto da ventotto anziani che, assieme ai due re, avevano i pieni poteri legislativi ${ }^{4}$; il biografo greco sottolinea inoltre come il popolo, all'interno delle assemblee, avesse in ogni caso il diritto di veto sulle leggi proposte dal consiglio. Tuttavia la cittadinanza, con il conseguente diritto di partecipazione attiva alla vita politica, era una prerogativa di pochi: nell'ordinamento spartano esistevano, oltre agli Spartiati (gli unici a godere dei pieni diritti), i Perieci, che abitavano all'interno del territorio laconico ma non nella stessa città di Sparta, i quali avevano diritto alla proprietà terriera ma non alla cittadinanza, e gli Iloti, privi di diritto alla cittadinanza e costretti a prestare servizio presso i proprietari terrieri.

Le stesse fonti classiche possono dare un'idea di quella che è, all'interno dello Stato spartano, la proporzione numerica tra Spartiati e non aventi diritto alla cittadinanza: Plutarco, parlando della divisione delle terre operata da Licurgo, afferma che il legislatore divise le terre della città di Sparta in novemila parti, da dividere tra gli Spartiati, $\mathrm{e}$ le restanti terre della Laconia in trentamila parti, da dividere tra i Periecis; inoltre Erodoto, parlando del contingente spartano inviato a prender parte alla battaglia di Platea, conta cinquemila Spartiati, ad ognuno dei quali erano assegnati sette Iloti ${ }^{6}$.

Riguardo al numero assoluto degli Spartiati, lo stesso Erodoto conta ottomila «uguali» (öpoıı, come solevano autodefinirsi gli Spartiati), mentre Aristotele, nella Politica, indica la quantità di diecimila come il massimo numerico da essi mai raggiunto ${ }^{8}$.

Su questi dati, evidentemente, Lévesque basa le proprie considerazioni sul sistema legislativo spartano, che effettivamente offre un valido termine di paragone con il sistema politico francese pre-rivoluzionario: il ricorso alla memoria storica del periodo merovingio e carolingio non va letto, alla lettera, come un confronto forzato tra la struttura oligarchica dello stato spartano ed il feudalesimo francese; si tratta di uno stratagemma, attraverso cui Lévesque lascia trasparire un'allusione a ben altri periodi della storia francese, caratterizzati da errori che lo stesso studioso stigmatizza, in maniera sempre velata, nei lavori in cui tratta delle dinamiche politico-culturali proprie della società spartana.

Nella sua opera Études de l'histoire ancienne et de celle de la Grèce, Lévesque dedica non poche pagine alla costituzione spartana, in cui non mancano aspre critiche a questo regime che, mascherato da una formale democrazia, si mostra in sostanza come un'oligarchia dura e spietata. Nel descrivere la forte disparità sociale esistente a Spar-

\footnotetext{
${ }^{4}$ Plutarco, Vita di Licurgo, V.

${ }^{5}$ Plutarco, Vita di Licurgo, VIII.

${ }^{6}$ Erodoto, Storie, IX, 10.

${ }^{7}$ Erodoto, Storie, VII, 234.

${ }^{8}$ Aristotele, Politica, II, 1270a.
} 
ta, 1'autore dichiara senza mezzi termini: «Quale libertà! quale uguaglianza! quando si vede un popolo diviso in due classi irrevocabilmente separate, di cui l'una ha dalla sua parte l'abbondanza e il potere, l'altra la più umile sottomissione e la miseria!» ${ }^{9}$. Di fatto, Lévesque cita opportunamente Plinio il Vecchio, che nel libro VII della sua Naturalis Historia, nel ricordare i contributi lasciati all'umanità da diversi personaggi e popoli antichi, definisce gli Spartani come coloro i quali «inventarono la servitù» ${ }^{10}$.

Altro aspetto a cui Lévesque dà molta importanza è la funzione sociale dei «sissi-

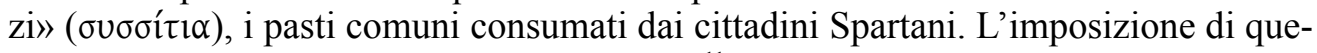
sto costume fu anch'essa attribuita a Licurgo ${ }^{11}$ : Plutarco ricorda che la stessa usanza vigeva a Creta, ma, mentre nell'isola questi pasti comuni venivano definiti $\alpha \nu \delta \rho \varepsilon \tilde{\alpha} \alpha$, accentuando il fatto che la partecipazione fosse riservata agli uomini, a Sparta si prefe-

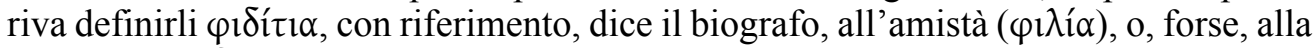
frugalità $(\varphi \varepsilon \imath \delta \omega)$ che li caratterizzava ${ }^{12}$; tuttavia, Aristotele fa notare che, se a Creta questi banchetti erano a spese pubbliche, a Sparta i cittadini erano obbligati a contribuire di tasca propria, il che andava a danno dei più poveri, i quali, nel caso in cui non riuscissero a supportare la spesa, perdevano il diritto di cittadinanza ${ }^{13}$.

Dunque, si chiede Lévesque, «come poteva la povertà essere debellata in una repubblica dove essa fu, si dice, stabilita dal legislatore?» ${ }^{14}$

Insistendo sul falso mito di una Sparta povera e poco interessata alla ricchezza, a cui lo studioso vuol sostituire la ben più realistica immagine di una città ricca ed ignorante, Lévesque cita un passo di Erodoto $(\mathrm{I}, 69)$, in cui viene menzionato l'oro ottenuto da Creso per la costruzione, nella città laconica di Tornax, di una statua del dio Apol$10^{15}$; ed ancora, menziona il decreto di "espulsione delle arti inutili" ( $\pi \varepsilon \rho \iota \sigma \sigma \tilde{\omega} \nu \tau \varepsilon \chi \vee \tilde{\omega} \nu$ $\xi \varepsilon \vee \eta \lambda \alpha \sigma i ́ \alpha)^{16}$, considerato da Plutarco come un ulteriore mezzo, ideato da Licurgo, per allontanare da Sparta il lusso e lo spreco, ma che Lévesque, al contrario, considera come un notevole contributo all'ignoranza e all'imbarbarimento della popolazione ${ }^{17}$.

Giungendo alla conclusione di questa parte dell'opera dedicata alla costituzione spartana, Lévesque attacca persino quell'aspetto divenuto emblema di una visione virtuosa dell'antica Sparta: il coraggio dei suoi cittadini. L'autore menziona un costume, probabilmente istituito dallo stesso Licurgo e tramandato da Plutarco con il nome di $\kappa \rho u \pi \tau \varepsilon i^{18}{ }^{18}$, che dava il diritto ai giovani Spartiati, in processo di formazione educativa, di andare per le campagne uccidendo a proprio piacimento gli Iloti; dunque,

${ }^{9}$ «Quelle liberté! quelle égalité! quand on voit un peuple divisé en deux classes irrévocablement séparées, dont l'une a pour son partage l'abondance et le pouvoir, l'autre la plus humble soumission et la misère!» (LÉVESQUE, Pierre-Charles (1811), II, p. 296).

${ }^{10}$ «Servitium invenere Lacedaemonii» (Plinio, Naturalis Historia, VII).

${ }^{11}$ Plutarco, Vita di Licurgo, X.

12 Plutarco, Vita di Licurgo, XII.

${ }^{13}$ Aristotele, Politica, II, 1271a.

${ }^{14}$ «Et comment la pauvreté pouvait-elle être punie dans une république où elle fut, dit-on, établie par le législateur?» (LÉvesQue, Pierre-Charles (1811), II, p. 310).

${ }^{15}$ LÉvesque, Pierre-Charles (1811), II, p. 311.

${ }^{16}$ Plutarco, Vita di Licurgo, IX.

${ }^{17}$ LÉVESQUe, Pierre-Charles (1811), II, p. 318.

${ }^{18}$ Plutarco, Vita di Licurgo, XXVIII. 
secondo Lévesque, formare dei giovani coraggiosi significava, a Sparta, nient'altro che «abituarli a versar sangue» ${ }^{19}$.

Anche nella sua traduzione della Guerra del Peloponneso di Tucidide, Lévesque non rinuncia all'aggiunta di commenti estremamente critici nei confronti dei costumi spartani; l'esempio più lampante è offerto dalle note con cui accompagna il celebre discorso funebre di Pericle: quando Tucidide, per bocca di Pericle, mette a confronto l'ospitalità e la colta educazione degli Ateniesi con l'eccessiva rigidità vigente a Sparta, Lévesque sottolinea il riferimento alle dure leggi spartane, che abituano i cittadini al disprezzo e al rifiuto degli elementi culturali provenienti dall'esterno, e definisce gli stessi Spartani «schiavi dei loro severi costumi» ${ }^{20}$.

Dunque diversi aspetti politico-legislativi, che avevano contribuito a creare un'immagine mitica dell'antica Sparta, vengono demoliti senza mezzi termini da Lévesque. Innanzitutto la pretesa «uguaglianza» a cui la definizione di ö $\mu$ oı doveva richiamare: definendosi «uguali», i cittadini spartani davano un'immagine del tutto fuorviante del proprio Stato, in cui, come ben nota Lévesque, il diritto ad essere «uguali», e dunque cittadini effettivi, era prerogativa di pochi, e dipendeva dalla ricchezza, in mancanza della quale lo stesso diritto si poteva perdere. Pertanto, denudando Sparta di una maschera fittizia, che le dava un falso aspetto sobrio e democratico, Lévesque mostra tra le righe l'inesattezza di chi, come l'abate filosofo Gabriel Bonnot de Mably, ha voluto vedere, nell'ordinamento di questa città, un egualitarismo che tutelasse i cittadini dal punto di vista giuridico, economico e sociale: da autentico precursore di un socialismo utopico, Mably elogiava la costituzione spartana come emblema della democrazia, vedendo, alla base di essa, un rifiuto assoluto del concetto di proprietà privata ${ }^{21}$. Chiaramente, la critica velata di Lévesque non poteva andare contro gli ideali di una giusta uguaglianza sociale, ma sicuramente contro il modello scelto per il rafforzamento degli stessi: lo studioso non nasconde mai il proprio risentimento nei confronti delle gravi ripetizioni dello stesso errore politico, che ha lasciato il potere ed i pieni diritti nelle mani di pochi, a danno della maggioranza della popolazione.

Tornando all'introduzione della sua edizione dei Detti dei Lacedemoni, ed in particolare al confronto operato tra la Francia delle dinastie merovingia e carolingia e lo Stato spartano, seppure la condanna si rivolga apertamente alla struttura sociale del feudalesimo medievale, appare chiara l'allusione a quegli aspetti sociali e politici che sopravvivono alla morte giuridica del feudalesimo, per l'annientamento dei quali la Francia dovrà aspettare la sua grande Rivoluzione. Tuttavia, la storia della Francia pre-rivoluzionaria non è il solo periodo a cui Lévesque fa allusione; evidentemente, attraverso la critica al regime spartano, lo studioso mostra il proprio disappunto nei confronti della fazione più radicale formatasi tra i rivoluzionari: i montagnardi, come sottolinea Vidal-Naquet, utilizzarono Sparta come strumento ideologico attraverso cui immaginare la nuova società francese, e non a caso lo stesso Robespierre dirà «Sparta brilla come un lampo in quest'immensa oscurità $\rangle^{22}$. La degenerazione ideologica che

\footnotetext{
${ }^{19}$ LÉvesque, Pierre-Charles (1811), II, p. 323.

${ }^{20}$ LÉVESQue, Pierre-Charles (1795b), I, p. 216.

${ }^{21}$ Bonnot de Mably, Gabriel (1768), lettera I.

${ }^{22}$ Vidal-NAQUet, Pierre (1992), p. 141.
} 
porterà alla dittatura del Terrore è, verosimilmente, l'autentico termine di paragone a cui Lévesque vuol fare cenno con la sua critica all'ordinamento spartano: i pochi «uguali» che formeranno parte del Comitato di Salute Pubblica saranno coloro i quali, senza prendere in considerazione le opinioni contrarie, governeranno per due anni un popolo francese represso e terrorizzato. Quando si pensa alla soppressione operata dalle «Colonne infernali» nella Vandea, in cui i soldati repubblicani, a seguito della rivolta antigiacobina, furono inviati ad uccidere qualunque vandeano incontrassero sul

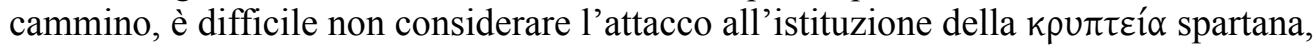
ferocemente stigmatizzata da Lévesque, come allusivo a quest'aberrante esempio di massacro istituzionalmente permesso.

In conclusione, la Sparta vista da Lévesque si mostra effettivamente come un «ritratto» della Francia pre-rivoluzionaria, ma non solo in senso storico: è la rivoluzione delle idee in campo sociale che, a quanto pare, agitava l'animo di questo studioso, il quale vedeva negli atteggiamenti filospartani non solo un errore di interpretazione storica, ma anche e soprattutto un regresso ad una logica antidemocratica, che accetta la maschera di un falso egualitarismo per dare sfogo ad un'incivile dittatura oligarchica. Il caso della lettura giacobina è l'emblema di questa falsa interpretazione della costituzione spartana e, in generale, delle esigenze di democrazia che invadevano la Francia di fine '700; non a caso, già prima dello scoppio della Rivoluzione francese, la scuola di pensiero filospartana fu sottoposta a dure critiche, tra cui emerge quella ad opera di Cornelius de Pauw: nelle sue Recherches philosophiques sur les Grecs, de Pauw non solo mise a nudo il vero carattere, squisitamente oligarchico, della costituzione spartana, ma pose soprattutto l'accento sul dato di fatto che, se pur si voleva parlare di democrazia, essa da un lato riguardava unicamente gli Spartiati, i quali formavano una minoranza elitaria, e dall'altro si presentava viziata dalla presenza istituzionale dei due re ${ }^{23}$.

Lévesque riesce ad andare al di là della semplice critica formale, mostrando, attraverso la condanna di un regime del passato, l'errore di chi considera democratico un ordinamento in cui l'uguaglianza sia diritto di una parte della popolazione, che acquisisce un potere attraverso il quale non dà garanzia di libertà a tutti; lo studioso lascia così uno spunto riflessivo di grande attualità, dimostrando che, in qualunque periodo storico, la vera morte della democrazia è provocata da coloro i quali intendono arrivare ad essa calpestando proprio quel diritto di uguaglianza assoluta che la genera.

${ }^{23}$ De Pauw, Cornelius (1787); GUERCI, Luciano (1979). 


\section{REFERENCIAS BIBLIOGRÁFICAS}

Bonnot de MABly, Gabriel (1768), Doutes proposés aux philosophes économistes sur l'ordre naturel et essentiel des sociétés politiques, La Haye.

De Feller, François-Xavier (1849), Biographie universelle, ou dictionnaire historique des hommes qui se sont fait un nom, Paris.

De Pauw, Cornelius (1787), Recherches philosophiques sur les Grecs, Berlin.

GuERCI, Luciano (1979), Libertà degli antichi e libertà dei moderni, Napoli.

LÉVESQue, Pierre-Charles (1794), Apophthegmes des Lacédémoniens, extraits de Plutarque, Paris.

- (1795), Vies et apophthegmes des philosophes grecs, Paris.

- (1795b), Histoire de Thucydide, Paris.

- (1811), Études de l'histoire ancienne et de celle de la Grèce, Paris.

VIDAL-NAQUeT, Pierre (1992), La democracia griega, una nueva visión, Madrid. 